\title{
Article
}

\section{Rheological and Dielectric Behavior of Polyisoprene under Pressurized Carbon Dioxide}

\author{
Akiko UnO, Yumi MatsumiYa*, Yuichi Masubuchi, and Hiroshi Watanabe \\ Institute for Chemical Research, Kyoto University, Uji, Kyoto 611-0011 Japan \\ (Received: January 5, 2010)
}

\begin{abstract}
For a well-entangled polyisoprene $(\mathrm{PI}$; molecular weight $=260 \mathrm{k})$ equilibrated under pressurized carbon dioxide $\left(\mathrm{CO}_{2}\right)$ at $25^{\circ} \mathrm{C}$, linear viscoelastic and dielectric data, respectively, were measured with a stress-controlled rheometer and a dielectric bridge being equipped with respective high-pressure cells. The viscoelastic and dielectric data shifted to higher frequencies with increasing $\mathrm{CO}_{2}$ pressure, indicating that the pressurized $\mathrm{CO}_{2}$ dissolved into PI thereby accelerating the global motion of PI. For those data at various $\mathrm{CO}_{2}$ pressure, time- $\mathrm{CO}_{2}$ pressure superposition held well and a single master curve was obtained, and the horizontal/vertical shift factors were consistent for the viscoelastic and dielectric data. These results indicated that the dissolved $\mathrm{CO}_{2}$ behaved just as an ordinal solvent to accelerate the global motion of PI as in ordinary solutions. In fact, the dynamic tube dilation (DTD) relationship between the viscoelastic and dielectric data, known to be valid for ordinary solutions/bulk of linear PI, was found to work also the PI/CO $\mathrm{C}_{2}$ system, which confirmed the simple solvent role of the pressurized $\mathrm{CO}_{2}$ for the global motion of PI. Thus, the knowledge for ordinary polymer solutions would work for processing of polymer $/ \mathrm{CO}_{2}$ systems.
\end{abstract}

Key Words: Pressurized carbon dioxide / Polyisoprene / Rheological and dielectric behavior

\section{INTRODUCTION}

Carbon dioxide $\left(\mathrm{CO}_{2}\right)$ is widely utilized in the field of polymer processing. ${ }^{1,2)}$ Pressurized $\mathrm{CO}_{2}$ is considerably soluble in a variety of polymers and decreases their glass transition temperature $\left(T_{\mathrm{g}}\right)$. For a practical purpose, it is important to know the polymer rheology under pressurized $\mathrm{CO}_{2}$. In fact, rheological measurements have been performed with pressure cells to reveal non-Newtonian flow behavior of polymers under fast shear. ${ }^{3-7)}$ It is also desired to investigate an effect of $\mathrm{CO}_{2}$ on the polymer dynamics at equilibrium, but just a few set of linear viscoelastic data under pressurized $\mathrm{CO}_{2}$ (for low density polyethylene ${ }^{8-10)}$ and polypropyrene ${ }^{9,10}$ ) has been reported due to the technical difficulties. ${ }^{8}{ }^{810}$ Those linear viscoelastic data were obtained with a stress rheometer equipped with a high-pressure cell, and only a limited aspect of the polymer dynamics has been elucidated because the magnetic driving mechanism of the cells works just in a rather narrow range of frequency.

For full investigation of the global dynamics of polymers governing the terminal relaxation/flow behavior at equilibrium, we recently conducted a dielectric test on polyisoprene

\footnotetext{
* to whom correspondence should be addressed.

E-mail: matsumiya@scl.kyoto-u.ac.jp, Tel: -81 774-38-3147, Fax: -81 774-38-3139
}

(PI) of a moderate molecular weight $\left(M_{\mathrm{w}}=2.0 \times 10^{4}\right)$ under pressurized $\mathrm{CO}_{2}$ up to the pressure of $P_{\mathrm{CO}_{2}}=10 \mathrm{MPa}$ at $65{ }^{\circ} \mathrm{C}$ (which included the supercritical state of $\mathrm{CO}_{2}$ ) and at frequencies ranging from $1 \mathrm{~Hz}$ to $10 \mathrm{MHz} .{ }^{11)} \mathrm{PI}$ has the type-A dipole parallel along the chain backbone and thus the dielectric data (easily measured even in a high pressure cell) reflected the end-to-end vector fluctuation of the PI chain, i.e., the motion over the maximum length scale of the chain. This motion was accelerated while its mode distribution was not affected by pressurized/dissolved $\mathrm{CO}_{2}$ even for the case of $\mathrm{CO}_{2}$ in the supercritical state. This result suggested that $\mathrm{CO}_{2}$ behaves as an ordinary solvent (or plasticizer) for the global dynamics of PI.

Even though the above dielectric results provided us a new insight for the role of the pressurized $\mathrm{CO}_{2}$ in the global dynamics of PI, it is still early to conclude that $\mathrm{CO}_{2}$ behaves as an ordinary solvent in all aspects. In fact, the effect of $\mathrm{CO}_{2}$ on the local segmental dynamics (acceleration without mode broadening) is known to be different from that of ordinary solvents (acceleration with mode broadening), and this difference is attributable to very rapid motion of the $\mathrm{CO}_{2}$ molecules. ${ }^{12)}$ Thus, it is desired to test if $\mathrm{CO}_{2}$ perfectly behaves as an ordinary solvent for the slow, global dynamics of polymers.

For this purpose, we can utilize a difference between the slow viscoelastic and dielectric relaxation processes of 
linear PI chains. These processes detect the same thermal motion of the chain in a different way and thus the frequency dependence (relaxation mode distribution) is totally different for the complex modulus $G^{*}$ and complex dielectric constant $\varepsilon^{*}:{ }^{13}$ The mode distributions of $G^{*}$ and $\varepsilon^{*}$ are sensitive and insensitive, respectively, to the entanglement. This difference results from the difference in the fundamental molecular functions of the viscoelastic and dielectric relaxation, the orientation function and local correlation function, the former being an isochronal, second-order average of the bond vectors $\mathbf{u}$ of the stress sustaining unit while the latter being the non-isochronal second-order average (average of $\mathbf{u}$ at different times). ${ }^{13)}$ Thus, the comparison of the viscoelastic and dielectric data for $\mathrm{PI} / \mathrm{CO}_{2}$ systems would enable us to experimentally test if $\mathrm{CO}_{2}$ really behaves as an ordinary solvent for the slow, global dynamics of PI.

This paper reports the results of this test. Because of the difference between the fundamental molecular functions of the viscoelastic and dielectric relaxation, there is no universal relationship between viscoelastic and dielectric data of PI having the type-A dipoles. However, for entangled linear PI, a simple relationship reflecting the finite lifetime of entanglements is known to hold between the normalized viscoelastic and dielectric relaxation functions. ${ }^{13,14)}$ Thus, we tested the validity of this relationship for the $\mathrm{PI} / \mathrm{CO}_{2}$ system. The relationship did hold for the $\mathrm{PI} / \mathrm{CO}_{2}$ system, confirming that $\mathrm{CO}_{2}$ behaves as an ordinary solvent/plasticizer for the global motion of entangled polymers. The details of this result are presented below.

\section{EXPERIMENTAL}

\subsection{Material}

Polyisoprene (PI260k, $M_{\mathrm{w}}=2.6 \times 10^{5}, M_{\mathrm{w}} / M_{\mathrm{n}}=1.7$ ) was kindly supplied from Sumitomo Rubber Industries, Co., and was used as received. Carbon dioxide $\left(\mathrm{CO}_{2}\right)$ was purchased from Kyoto Teisan Co. Ltd. and used as received.

\subsection{Dielectric Measurement}

The setup and the protocol of the dielectric measurements under pressurized $\mathrm{CO}_{2}$ were basically the same as those reported in our previous studies: ${ }^{11,12)}$ The sample (PI260k) was charged between main and counter electrodes made of sintered metal (the former having a guard electrode) placed in a highpressure jacket, exposed to $\mathrm{CO}_{2}$ having the pressure $P_{\mathrm{CO}_{2}} / \mathrm{MPa}$ $=0$ (atmospheric ambient pressure), 2, 4, 6, 8, and 10 and fully equilibrated (as monitored through lack of the time dependent change of the dielectric data), and then subjected to the measurement at each $P_{\mathrm{CO}_{2}}$. All these operations were made at $25^{\circ} \mathrm{C}$, and the measurement was made in the increasing order of $P_{\mathrm{CO}_{2}}$. The vapor pressure of $\mathrm{CO}_{2}$ at $25{ }^{\circ} \mathrm{C}$ is 6.8 MPa. ${ }^{15)}$ Therefore, $\mathrm{CO}_{2}$ was in gaseous state at $P_{\mathrm{CO}_{2}} \leq 6 \mathrm{MPa}$ while in liquid state at $P_{\mathrm{CO}_{2}}=8$ and $10 \mathrm{MPa}$.

The dielectric signal was measured with an impedance analyzer combined with a dielectric interface (1260/1296, Solartron) in a range of the angular frequency $\omega$ between $10^{-2} \mathrm{~s}^{-1}$ to $10^{4} \mathrm{~s}^{-1}$. Differing from the previous study, we utilized a $\mathrm{CO}_{2}$ pressure controlling system, a syringe-type pump (260D, Isco) with flow panel, which was shared with the rheological setup explained later. This system enabled a little better control of the $\mathrm{CO}_{2}$ pressure compared to the previous setup $^{11,12)}$ (having direct connection to a $\mathrm{CO}_{2}$ tank through a regulator).

\subsection{Linear Viscoelastic Measurement}

A stress-controlled rheometer (MCR-301, Anton Paar) equipped with a high-pressure cell unit (Anton Paar) was employed for the linear viscoelastic measurements. $\mathrm{CO}_{2}$ cylinder with a gas regulator was connected to a syringetype pump (260D, Isco) equipped with a flow control panel, which allowed the pressured $\mathrm{CO}_{2}$ gas to be introduced to the high-pressure cell. Figure 1 illustrates the measurement setup including this cell. The sealed cell has a gas inlet that allows $\mathrm{CO}_{2}$ gas to be introduced. The sample is placed between the lower fixture $(25 \mathrm{~mm} \phi)$ and the upper plate-type $(20 \mathrm{~mm} \phi)$ fixture, the latter being made of a sintered metal and having a magnet on its top part. The upper fixture was driven by the outside magnet to apply a commanded stress (torque) to the specimen, and the corresponding strain was detected. With this driving mechanism, the measurements could not be well conducted at high angular frequencies $\omega$ (where the

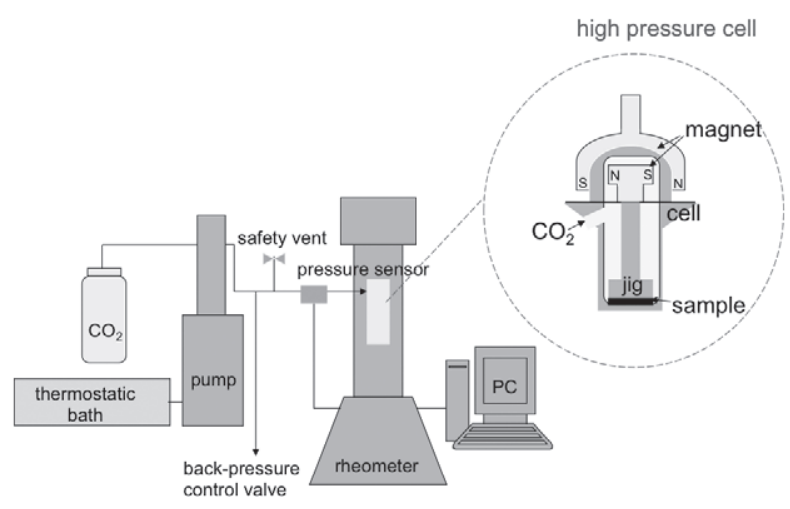

Fig. 1. Schematic illustration of viscoelastic measurement setup including the high-pressure cell. 
upper fixture cannot follow the outside magnet to exhibit a mechanical delay) and at low $\omega$ (where the friction disturbed an accurate application of the torque), as reported previously. Thus, we utilized an ordinary, directly-driven strain-controlled rheometer (ARES, Rheometrics) to calibrate/correct the highpressure setup of the stress-controlled rheometer with the previous method ${ }^{8)}$ (the calibration/correction being made for the inertia/friction of the upper fixture and the difference of the upper and lower fixture diameters) and specify the range of $\omega\left(=10^{-1} \mathrm{~s}^{-1}-10 \mathrm{~s}^{-1}\right)$ where we could reliably utilize the high-pressure setup. The data of storage and loss moduli, $G^{\prime}$ and $G^{\prime \prime}$, obtained after the calibration/correction are believed to be acceptably accurate (with uncertainty smaller than $30 \%$, as judged from reproducibility in several independent runs) and the best available data at this moment.

The measurement was conducted for PI260k under $\mathrm{CO}_{2}$ in the high-pressure setup at $25^{\circ} \mathrm{C}$. The $\mathrm{CO}_{2}$ pressure was chosen to be $P_{\mathrm{CO}_{2}} / \mathrm{MPa}=0,2,4,6,8$, and 10 , and the equilibration of PI260k (saturation with $\mathrm{CO}_{2}$ ) at each $P_{\mathrm{CO}_{2}}$ was confirmed from lack of time-dependent change of the moduli (at $\omega=6.28 \mathrm{~s}^{-1}$ ) for the exposure time $\geq 15 \mathrm{~h}$. The experimental run at each equilibrated state was made in the order of increasing $P_{\mathrm{CO}_{2}}$.

\section{RESULTS}

\subsection{Dielectric Behavior of $\mathrm{PI} 260 \mathrm{k}$ under pressurized $\mathrm{CO}_{2}$}

Figures 2(a) and 2(b), respectively, show the angular frequency $(\omega)$ dependence of the decease of dielectric constant, $\varepsilon_{0}-\varepsilon^{\prime}(\omega)$ with $\varepsilon_{0}=\varepsilon^{\prime}(0)$, and the dielectric loss, $\varepsilon^{\prime \prime}(\omega)$, of PI260k equilibrated under various $P_{\mathrm{CO}_{2}}$ at $25^{\circ} \mathrm{C}$. (In the phenomenological framework of linear stimulusresponse theory, $\varepsilon_{0}-\varepsilon^{\prime}(\omega)$ and $\varepsilon^{\prime \prime}(\omega)$ are analogous to the viscoelastic $G^{\prime}$ and $G^{\prime \prime}$, respectively, and expressed in terms
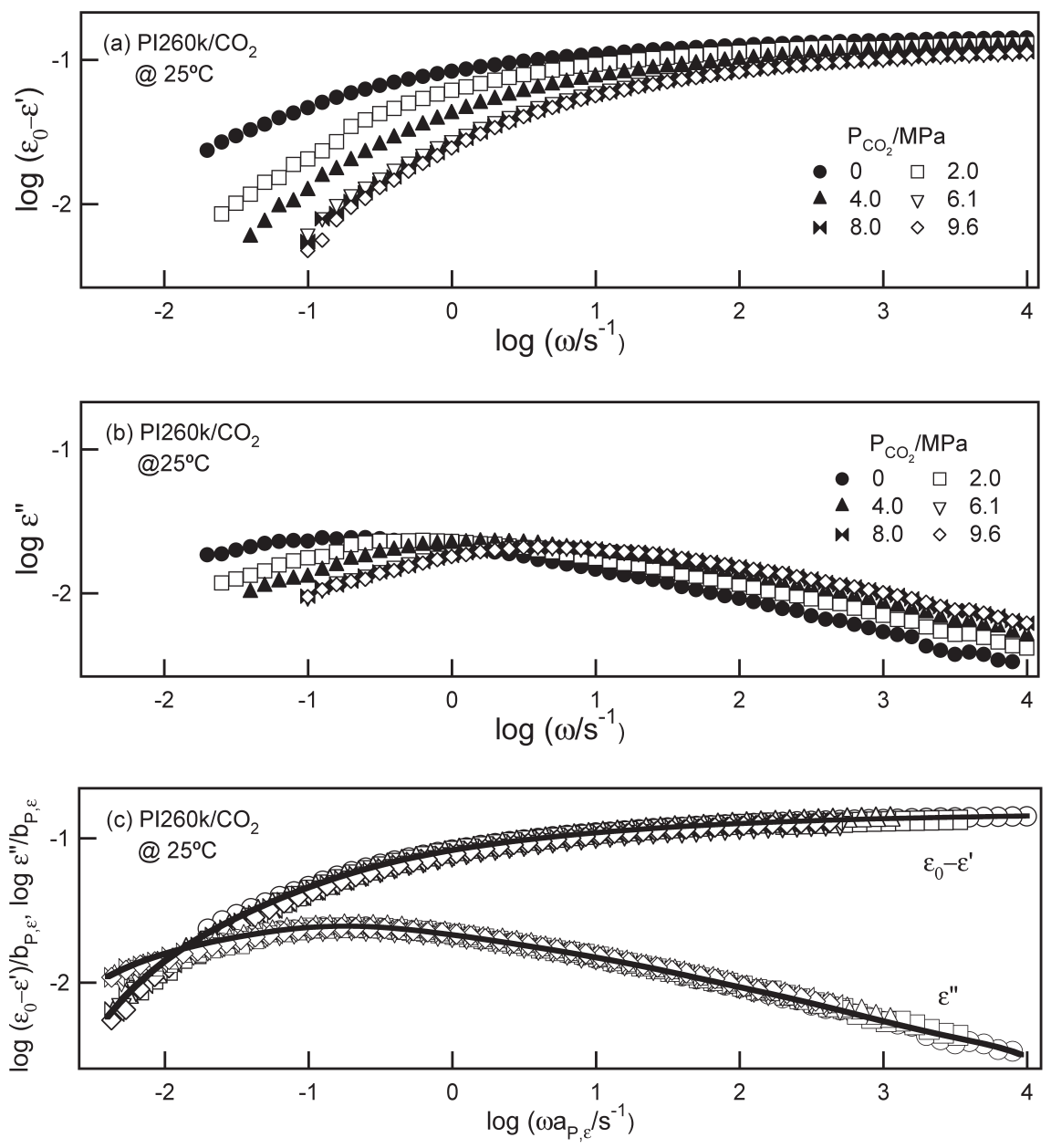

Fig. 2. Frequency dependence of (a) decrease of dielectric constant, $\varepsilon_{0}-\varepsilon^{\prime}$, and (b) dielectric loss, $\varepsilon^{\prime \prime}$, measured for PI260k equilibrated under various $P_{\mathrm{CO}_{2}}$ at $25^{\circ} \mathrm{C}$. Panel (c) shows the time- $\mathrm{CO}_{2}$ pressure superposed master curve of the $\varepsilon_{0}-\varepsilon^{\prime}$ and $\varepsilon^{\prime \prime}$ data. Solid curves indicate $\varepsilon_{0}-\varepsilon^{\prime}$ and $\varepsilon^{\prime \prime}$ recalculated from eq. 8 with the normalized dielectric intensities $g_{\mathrm{p}}$ and dielectric relaxation times $\tau_{\mathrm{p}}$ therein being determined from an iterative fitting method. ${ }^{21)}$ For more details, see text. 
of the relaxation spectrum. ${ }^{13)}$ ) $\mathrm{CO}_{2}$ is a non-polar molecule and is dielectrically inert at $\omega$ examined. Hence the dielectric relaxation observed here is exclusively attributed to the global motion of well entangled PI260k chains. This relaxation shifts toward higher $\omega$ and its intensity decreases with increasing $P_{\mathrm{CO}_{2}}$ up to $6 \mathrm{MPa}$, which indicates that the pressurized $\mathrm{CO}_{2}$ dissolves in PI260k to give the plasticization and dilution effects on the global motion at those $P_{\mathrm{CO}_{2}}$. A similar result was noted also in our previous study. ${ }^{11)}$ In contrast, at $P_{\mathrm{CO}_{2}} \geq 8 \mathrm{MPa}$ exceeding the vapor pressure of $\mathrm{CO}_{2}$ at $25^{\circ} \mathrm{C}\left(=6.8 \mathrm{MPa}^{15)}\right)$, the $\varepsilon_{0}-\varepsilon^{\prime}$ and $\varepsilon^{\prime \prime}$ data become insensitive to $P_{\mathrm{CO}_{2}}$ possibly because the dissolution of $\mathrm{CO}_{2}$ into PI was saturated due to liquefaction of $\mathrm{CO}_{2}$.

Concerning this behavior at high $P_{\mathrm{CO}_{2}}$, a reviewer of this paper suggested a possibility of liquid-liquid phase separation of PI being equilibrated with liquefied $\mathrm{CO}_{2}$. This phase separation should unavoidably lead to coexistence of slow and fast PI chains (in PI-rich and PI-poor phases, respectively). Nevertheless, no dielectric mode broadening attributable to this coexistence is observed in Figure 2, which indicates that our PI equilibrated with liquefied $\mathrm{CO}_{2}$ exhibited negligible liquid-liquid phase separation and stayed in uniform, one phase state even under the high $P_{\mathrm{CO}_{2}}$ examined.

Figure 2(c) shows the time- $P_{\mathrm{CO}_{2}}$ superposed master curves, $\left(\varepsilon_{0}-\varepsilon^{\prime}\right) / b_{\mathrm{p}, \varepsilon}$ and $\varepsilon^{\prime \prime} / b_{\mathrm{p}, \varepsilon}$ as a function of $\omega a_{\mathrm{p}, \varepsilon} / \mathrm{s}^{-1}$. The shift factors, $a_{\mathrm{p}, \varepsilon}$ and $b_{\mathrm{p}, \varepsilon}$ are shown later in Figures 4(a) and 4(b). The excellent superposition seen in Figure 2(c) indicates that $\mathrm{CO}_{2}$ does not affect the dielectric relaxation mode distribution and thus has no influence for the mode of chain motion (relaxation mechanism) of the entangled PI260k. A similar result was noted in our previous study. ${ }^{11)}$

\subsection{Viscoelastic behavior of $\mathrm{Pl} 260 \mathrm{k}$ at pressurized $\mathrm{CO}_{2}$}

Figures 3(a) and 3(b), respectively, show storage and loss moduli, $G^{\prime}(\omega)$ and $G^{\prime \prime}(\omega)$, of PI260k equilibrated under various $P_{\mathrm{CO}_{2}}$ at $25^{\circ} \mathrm{C}$. The $G^{\prime}$ and $G^{\prime \prime}$ curves shift toward higher frequencies with increasing $P_{\mathrm{CO}_{2}}$ up to $6 \mathrm{MPa}$, which reflects the plasticization and dilution effects of unliquefied $\mathrm{CO}_{2}$. For $P_{\mathrm{CO}_{2}} \geq 8 \mathrm{MPa}$, the $G^{\prime}$ and $G^{\prime \prime}$ curves become almost insensitive to $P_{\mathrm{CO}_{2}}$ due to saturation of dissolved $\mathrm{CO}_{2}$ concentration on liquefaction of $\mathrm{CO}_{2}$. These features are qualitatively similar to those seen for the dielectric data (Figures 2(a) and 2(b)).

For the $G^{\prime}$ and $G^{\prime \prime}$ data, we tested the time- $\mathrm{CO}_{2}$ pressure superposition. The results are shown in Figure 3(c). Clearly, the superposition holds for those data, again indicating that $\mathrm{CO}_{2}$ does not affect the mode of chain motion (relaxation mechanism) of the entangled PI260k. The shift factors for the modulus data, $a_{\mathrm{p}, \mathrm{G}}$ and $b_{\mathrm{p}, \mathrm{G}}$ are compared with the dielectric shift factors, $a_{\mathrm{p}, \varepsilon}$ and $b_{\mathrm{p}, \varepsilon}$, later in Figures $4(\mathrm{a})$ and $4(\mathrm{~b})$.

\section{DISCUSSION}

\subsection{Shift Factors}

Figures 4(a) and 4(b), respectively, show the dielectric/ viscoelastic horizontal shift factors, $a_{\mathrm{P}, e}$ and $a_{\mathrm{P}, \mathrm{G}}$, and dielectric/ viscoelastic vertical shift factors, $b_{\mathrm{P}, \varepsilon}$ and $b_{\mathrm{P}, \mathrm{G}}$, utilized in Figures 2(c) and 3(c). These shift factors strongly depend on $P_{\mathrm{CO}_{2}}$ in the range of $P_{\mathrm{CO}_{2}}<6 \mathrm{MPa}$ (where $\mathrm{CO}_{2}$ has not liquefied) while the $P_{\mathrm{CO}_{2}}$ dependence becomes weak for $P_{\mathrm{CO}_{2}} \geq 8 \mathrm{MPa}$ (where liquefaction occurs), as already noted in Figures 2 and 3.

The dielectric, horizontal and vertical shift factors, $a_{\mathrm{P}}$ and $b_{\mathrm{P}}$, respectively are related to the acceleration of the PI chain
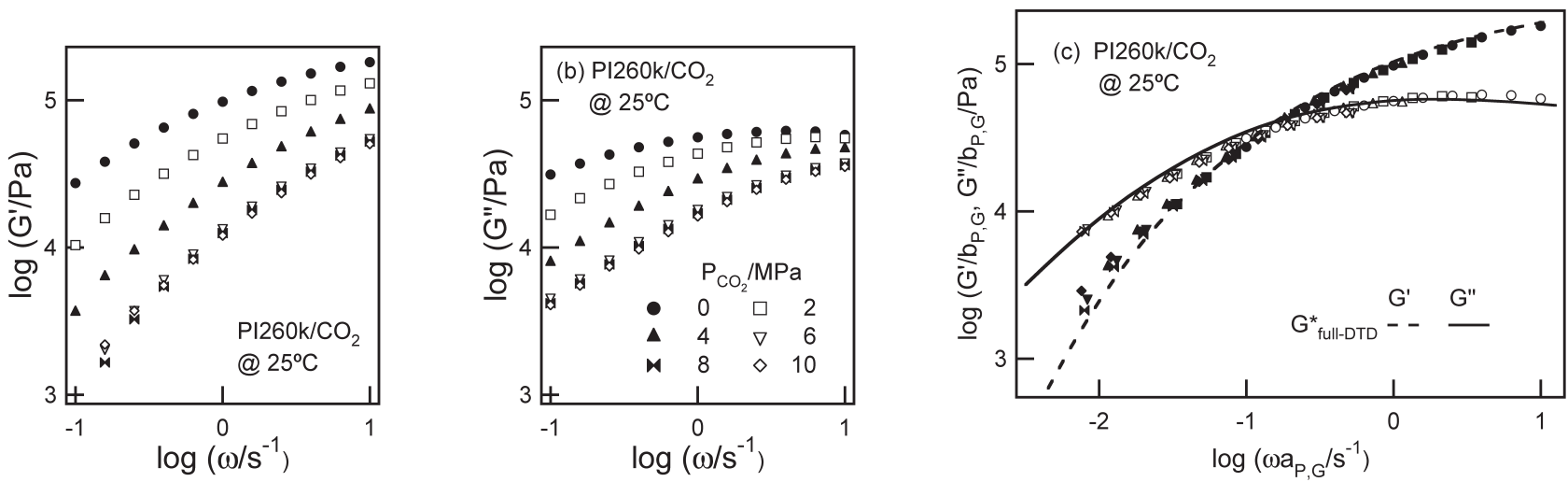

Fig. 3. Linear viscoelastic storage and loss moduli, (a) $\mathrm{G}^{\prime}$ and (b) $\mathrm{G}^{\prime \prime}$, measured for PI260k equilibrated under various $P_{\mathrm{CO}_{2}}$ at $25{ }^{\circ} \mathrm{C}$. Panel (c) shows the time- $\mathrm{CO}_{2}$ pressure master curve of the moduli data. Dashed and solid curves in panel (c) show $G^{\prime}$ and $G^{\prime \prime}$ calculated from the dielectric data (Figure 2) through the full-DTD relationship, eq 6. 
motion and dilution (decrease of the PI concentration) on the increase of $P_{\mathrm{CO}_{2}}$, as noted in our previous work. ${ }^{11)}$ The viscoelastic horizontal shift factor $a_{\mathrm{P}, \mathrm{G}}$ agrees well with the dielectric $a_{\mathrm{P}, \varepsilon}$, indicating that the viscoelastic and dielectric data commonly detect the accelerated PI chain motion under $\mathrm{CO}_{2}$. On the other hand, the dielectric/viscoelastic vertical shift factors, $b_{\mathrm{P}, \varepsilon}$ and $b_{\mathrm{P}, \mathrm{G}}$, are considerably different from each other. This difference can be related to the entanglement, as discussed below.

The dielectric $b_{\mathrm{P}, \varepsilon}$ factor is directly related to the concentration/ volume fraction of $\mathrm{PI}$ in the $\mathrm{PI} / \mathrm{CO}_{2}$ system, as clearly noted from the expression of the dielectric intensity $\Delta \varepsilon,{ }^{16,17)}$

$$
\Delta \varepsilon=F g v \frac{p^{2}}{3 k_{\mathrm{B}} T}
$$

Here, $F$ is Onsagar factor having a value of the order of unity, $p$ is the total dipole moment of a molecule (of the PI chain), $k_{\mathrm{B}}$ is the Boltzmann constant, $T$ is the absolute temperature, and $v$ is the number density of the dipoles. $g$ is the KirkwoodFröhlich factor that represents motional correlation of the dipoles. Since the dielectric mode distribution of PI260k does not change with $P_{\mathrm{CO}_{2}}$ (cf. Figure 2), the dielectric vertical shift factor $b_{\mathrm{P}, \varepsilon}$ is identical to a ratio of the of the dielectric intensity $\Delta \varepsilon$ of $\mathrm{PI}$ in the presence of the pressurized $\mathrm{CO}_{2}$ to that of neat (bulk) PI in a reference state at $P_{\mathrm{CO}_{2}}=0$. We may safely assume that $F$ and $g$ are insensitive to the existence $\mathrm{CO}_{2}{ }^{11)}$ and that the total dipole moment per PI chain, $p$, is constant given that the chain is in the concentrated state where the excluded volume effect is negligible. Then, from eq $1, b_{\mathrm{P}, \varepsilon}$ is expressed in terms of the chain number density $v$ as

$$
b_{\mathrm{P}, \varepsilon}=\frac{v}{v_{\mathrm{ref}}}=\frac{\Delta \varepsilon}{\Delta \varepsilon_{\mathrm{ref}}}
$$

Note that this expression of $b_{\mathrm{P}, \varepsilon}$ is not affected by the entanglement. ${ }^{11)}$ The $b_{\mathrm{P}, \varepsilon}$ data (Figure 4(b)) indicate that the $v /$ $v_{\text {ref }}$ ratio decreases only to $\sim 0.85$ with increasing $P_{\mathrm{CO}_{2}}$ up to 10 MPa. Thus, the above assumption of the lack of the excluded volume effect is consistent with the $b_{\mathrm{P}, \varepsilon}$ data.

Since the viscoelastic mode distribution of PI260k does not change with $P_{\mathrm{CO}_{2}}$ (cf. Figure 2), the viscoelastic vertical shift factor $b_{\mathrm{P}, \varepsilon}$ of the entangled PI260k is equivalent to a ratio of the plateau modulus $G_{\mathrm{N}}$ in the presence of the pressurized $\mathrm{CO}_{2}$ to that of neat (bulk) PI in a reference state at $P_{\mathrm{CO}_{2}}=0$. Thus, the $b_{\mathrm{P}, \mathrm{G}}$ factor is affected by the entanglement, as noted from the well known expression of $G_{\mathrm{N}}{ }^{13,18)}$

$$
G_{\mathrm{N}}=\frac{v M}{M_{\mathrm{e}}} k_{\mathrm{B}} T
$$

where $M$ is the molecular weight of the chain and the product $v M$ represents the mass concentration, and $M_{\mathrm{e}}$ is the molecular weight between entanglements. $M_{\mathrm{e}}$ in concentrated solutions is related to $M_{\mathrm{e}}{ }^{\circ}$ in bulk and the volume fraction of the polymer $v$ as

$$
M_{\mathrm{e}}=\frac{M_{\mathrm{e}}^{\mathrm{o}}}{v^{\alpha}}
$$

where $\alpha$ is the dilation exponent; $\alpha \cong 1.3$ for monodisperse linear PI. ${ }^{19)}$ Thus, the plateau modulus $G_{\mathrm{N}}$ scales as $v^{1+\alpha}$, not $v^{1}$, which results in the entanglement effect on the viscoelastic $b_{\mathrm{P}, \mathrm{G}}$ of the well entangled PI260k $\left(b_{\mathrm{P}, \mathrm{G}}=G_{\mathrm{N}} / G_{\mathrm{N}}{ }^{0}=v^{1+\alpha}\right)$. The difference between the dielectric $b_{\mathrm{P}, \varepsilon}\left(=v / v_{\text {ref }}\right)$ and the viscoelastic $b_{\mathrm{P}, \mathrm{G}}$ quite possibly reflects this entanglement
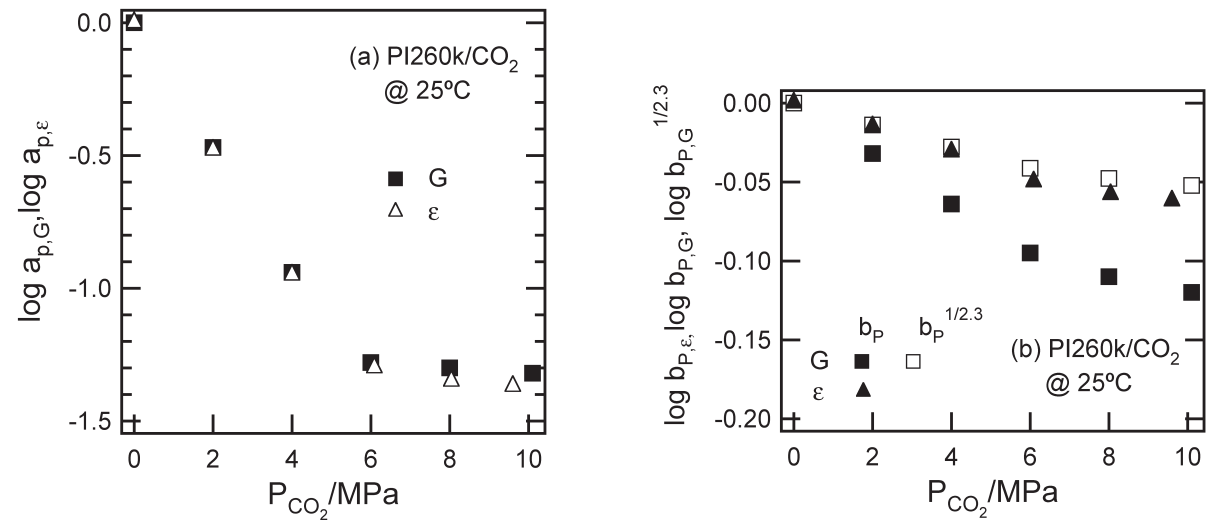

Fig. 4. $\mathrm{CO}_{2}$ pressure dependence of (a) viscoelastic/dielectric horizontal shift factors, $a_{\mathrm{P}, \mathrm{G}}$ (filled square) and $a_{\mathrm{P}, \varepsilon}$ (open triangle), and (b) viscoelastic/ dielectric vertical shift factors, $b_{\mathrm{P}, \mathrm{G}}$ (filled square) and $b_{\mathrm{P}, \varepsilon}$ (filled triangle), obtained for PI260k equilibrated under various $P_{\mathrm{CO}_{2}}$ at $25{ }^{\circ} \mathrm{C}$. The unfilled square in panel (b) indicates plots of $b_{\mathrm{P}, \mathrm{G}}^{1 / 2.3}$. For further details, see text. 
effect. In fact, the volume change on mixing PI and $\mathrm{CO}_{2}$ can be safely neglected and $v$ is well approximated as $v / v_{\text {ref }}$, which suggests a simple relationship, $b_{\mathrm{P}, \varepsilon}=b_{\mathrm{P}, \mathrm{G}}^{1 /(1+\alpha)}$ with $\alpha \cong 1.3$. This relationship holds within experimental uncertainty (compare the plots of $b_{\mathrm{P}, \varepsilon}$ and $b_{\mathrm{P}, \mathrm{G}}^{1 / 2.3}$ in Figure 4(b)), confirming the basic validity of our argument.

\subsection{Relationship Between Dielectric and Viscoelastic Relaxation Functions}

The global dynamics of entangled linear PI chain is well described by dynamic tube dilation (DTD) mechanism within the framework of the current tube model. ${ }^{13,17,19)}$ For monodisperse, entangled linear PI in bulk, the terminal relaxation has been found to be well described by the fullDTD picture that is intimately related to the double reptation picture: ${ }^{13,17,19)}$ In the full-DTD picture, the relaxed portions of the chains are regarded as a simple solvent having no contribution to the entanglement, and the normalized viscoelastic and dielectric relaxation functions, $\mu(t)$ $\left(=G(t) / G_{\mathrm{N}}\right)$ and $\Phi(t)$, are related to the survival fraction of the dilating tube $\varphi^{\prime}(t)$ as ${ }^{19)}$

$$
\begin{aligned}
& \mu(t)=\left\{\varphi^{\prime}(t)\right\}^{1+\alpha} \text { with } \alpha=1.3 \text { for PI } \\
& \Phi(t)=\varphi^{\prime}(t)+\text { tube edge fluctuation term }
\end{aligned}
$$

The "tube edge fluctuation term" in eq $5 b$ indicates the dielectric memory decay due to lateral fluctuation of the chain within the dilating tube. Equations $5 \mathrm{a}$ and $5 \mathrm{~b}$ specify the fullDTD relationship between the viscoelastic $\mu(t)$ and $\Phi(t)$ data that has been confirmed experimentally. ${ }^{19)}$ This relationship is numerically very close to a simple power-law relationship, ${ }^{19}$

$$
\mu(t)=\{\Phi(t)\}^{2}
$$

Thus, we can test the validity of eq 6 for our $\mathrm{PI} / \mathrm{CO}_{2}$ system to examine if the $\mathrm{PI} / \mathrm{CO}_{2}$ system behave as the ordinary solution thereby allowing the mode of global motion of PI therein to remain unaffected by $\mathrm{CO}_{2}$ and coincide with that of bulk PI.

A comment needs to be made for this test. Differing from the monodisperse PI utilized in the previous test, ${ }^{13,17,19)}$ our PI has a rather broad, unimodal molecular weight distribution $\left(M_{\mathrm{w}} / M_{\mathrm{n}}=1.7\right)$. However, this unimodal distribution enhances the validity of the molecular picture of full-DTD (and double reptation). ${ }^{20)}$ Thus, the test of eq 6 unequivocally allows us to examine if the $\mathrm{PI} / \mathrm{CO}_{2}$ system behave as the ordinary solution.

For the test of eq 6, we can express the normalized dielectric relaxation function $\Phi(t)$ as a sum of dielectric relaxation modes,

$$
\Phi(t)=\sum_{p} g_{p} \exp \left(-\frac{t}{\tau_{p}}\right) \text { with } \sum_{p} g_{p}=1
$$

where $\tau_{p}$ and $g_{p}$ represent the relaxation time and normalized intensity of $p$-th mode. The corresponding expression of the data for the decease of dielectric constant, $\varepsilon_{0}-\varepsilon^{\prime}(\omega)$, and dielectric loss, $\varepsilon^{\prime \prime}(\omega)$, is given by ${ }^{17)}$

$$
\frac{\varepsilon_{0}-\varepsilon^{\prime}(\omega)}{\Delta \varepsilon}=\sum_{p} g_{p} \frac{\omega^{2} \tau_{p}{ }^{2}}{1+\omega^{2} \tau_{p}{ }^{2}}, \frac{\varepsilon^{\prime \prime}(\omega)}{\Delta \varepsilon}=\sum_{p} g_{p} \frac{\omega \tau_{p}}{1+\omega^{2} \tau_{p}{ }^{2}}(8)
$$

where $\Delta \varepsilon$ is the dielectric intensity. Applying a previously introduced iterative fitting method ${ }^{21)}$ to the $\varepsilon_{0}-\varepsilon^{\prime}(\omega)$ and $\varepsilon^{\prime \prime}$ $(\omega)$ data, we evaluated $\tau_{p}$ and $g_{p}$ appearing in eq 8 . The solid curves in Figure 2(c) shows $\varepsilon_{0}-\varepsilon^{\prime}(\omega)$ and $\varepsilon^{\prime \prime}(\omega)$ re-calculated from these $\tau_{p}$ and $g_{p}$. Excellent agreement between the curves and the data indicates satisfactory accuracy of the $\tau_{p}$ and $g_{p}$ obtained from the iterative fitting.

If eq 6 for the full-DTD picture is valid, the complex shear modulus $G^{*}(\omega)=G^{\prime}(\omega)+i G^{\prime \prime}(\omega)$ should be expressed in terms of the dielectric $\tau_{p}$ and $g_{p}$ determined as above:

$$
\frac{G^{\prime}(\omega)+i G^{\prime \prime}(\omega)}{G_{N}}=\sum_{p, q} h_{p q} \frac{\omega^{2} \tau_{p q}{ }^{2}+i \omega \tau_{p q}}{1+\omega^{2} \tau_{p q}{ }^{2}}
$$

$$
h_{p q}=g_{p} g_{q}, \tau_{p q}=\left[\tau_{p}^{-1}+\tau_{q}^{-1}\right]^{-1}
$$

The $G^{\prime}(\omega)$ and $G^{\prime \prime}(\omega)$ calculated from eq 9 are shown in Fig. 3(c) with the dashed and solid curves. These calculated curves excellently agree with the $G^{\prime}(\omega)$ and $G^{\prime \prime}(\omega)$ data, indicating that full-DTD relationship holds for $\mathrm{PI} / \mathrm{CO}_{2}$ systems. This result allows us to conclude that the PI260k/ $\mathrm{CO}_{2}$ system is nothing different from linear PI solutions in ordinary solvents (that obey the full-DTD picture) as long as $\mathrm{CO}_{2}$ pressure is not extremely high (to give a densification effect). Thus, conventional knowledge for ordinary polymer solutions would work for polymer processing with the aid of pressurized $\mathrm{CO}_{2}$.

\section{CONCLUSION}

We have conducted viscoelastic and dielectric measurements for entangled linear PI equilibrated under various $\mathrm{CO}_{2}$ pressures. $\mathrm{CO}_{2}$ dissolved into PI with increasing $\mathrm{CO}_{2}$ pressure to accelerate the global motion of PI observed as the viscoelastic and dielectric relaxation. The time $-\mathrm{CO}_{2}$ pressure superposition worked for the viscoelastic and dielectric data, and the shift factors for the two set of data were consistent to 
each other. The dynamic tube dilation relationship between the dielectric and viscoelastic data, being valid for ordinary solutions/bulk of entangled linear PI, was found to hold for the $\mathrm{PI} / \mathrm{CO}_{2}$ system. This result suggests that $\mathrm{CO}_{2}$ behaves as an ordinary solvent/plasticizer for PI and has no special effect on global dynamics of PI chain.

\section{Acknowledgment}

The authors would like to thank Professor Tadashi Inoue at Department of Macromolecular Science, Graduate School of Science, Osaka University, for his valuable comments and for his generous help for the experiments utilizing the highpressure dielectric cell. They also thank Mr. Haruo Shikuma at Department of Chemical Engineering, Kyoto University, for his help for the viscoelastic measurements with the highpressure cell. This work was partly supported by Grant-inAid for Scientific Research on Priority Area "Soft Matter Physics" and by Grant-in-Aid for Young scientists (B) from the Ministry of Education, Culture, Sports, Science and Technology, Japan (No. 18068009 and 22750204).

\section{REFERENCES}

(1) Tomasko DL, Li HB, Liu DH, Han XM, Wingert MJ, Lee LJ, Koelling KW, Ind. Eng. Chem. Res. 2003, 42, 6431-6456.

(2) Nalawade SP, Picchioni F, Janssen L, Progress in Polymer Science 2006, 31, 19-43.

(3) Royer JR, Gay YJ, Desimone JM, Khan SA, J Polym Sci Pol Phys 2000, 38, 3168-3180.

(4) Kwag C, Manke CW, Gulari E, J Polym Sci Polym Phys, 1999, 37, 2771-2781.
(5) Areerat S, Nagata T, Ohshima M. Polym Eng Sci 2002, 42, 2234-2245.

(6) Park HE, Dealy JM, Macromolecules 2006, 39, 5438-5452.

(7) Park HE, Lim ST, Laun HM, Dealy JM, Rheol. Acta 2008, 47, 1023-1038.

(8) Ouchi S, Masubuchi Y, Shikuma H, Int. Polym. Process. 2008, 23, 173-177.

(9) Shikuma H, Kihara S, Oshima M, Proceedings of Annual Meeting of JSPP, 2005, I-106.

(10) Shikuma H, Kihara S, Oshima M, Proceedings of JSPP Symposia, 2005, A-110.

(11) Matsumiya Y, Inoue T, Watanabe H, Kihara S, Ohshima M. Nihon Reoroji Gakkaishi (J. Soc. Rheol. Japan) 2007, 35, 155-161.

(12) Matsumiya Y, Inoue T, Iwashige T, Watanabe H. Macromolecules 2009, 42, 4712-4718.

(13) Watanabe H, Prog. Polym. Sci. 1999, 24, 1253-1403.

(14) Matsumiya Y, Watanabe H, Osaki K, Macromolecules 2000, 33, 499-506.

(15) Atkins P, de Paula J, Physical Chemistry $7^{\text {th }}$ ed. ,W.H. Freeman and Co., New York, 2002, Chapter 6, p. 139.

(16) Riande E, Saiz E, "Dipole Moments and Birefringence of Polymers", Prentice Hall, New Jersey, 1992.

(17) Watanabe H, Macromolecular Rapid Communications, 2001, 22, 127-175.

(18) Ferry JD, "Viscoelastic Properties of Polymers", $3^{\text {rd }}$ ed., Wiley, New York, 1980

(19) Watanabe H, Ishida S, Matsumiya Y, Inoue T, Macromolecules 2004, 37, 1937-1951.

(20) Milner S, J Rheol 1996, 40, 303-315.

(21) Watanabe H, Matsumiya Y, Inoue T, Macromolecules 2002, 35, 2339-2357. 